Pacific Journal of Mathematics

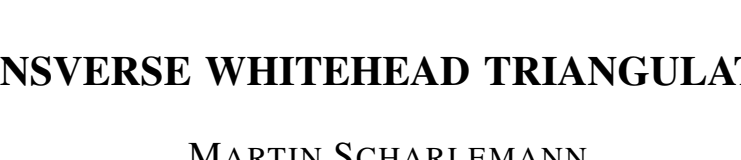




\section{TRANSVERSE WHITEHEAD TRIANGULATIONS}

\section{MARTIN SCHARLEMANN}

Suppose $M$ and $N$ are $P L$ manifolds and $f: M \rightarrow N$ is a proper $P L$ map. Triangulate $M$ and $N$ so that $f$ is simplical and let $X$ be the dual complex in $N$. Then for each open simplex $\sigma$ in $X, f^{-1}(\sigma)$ is a $P L$ submanifold of $M$, so the stratification of $N$ by the open simplices of $X$ pulls back to a stratification of $M$. In other words, any such $P L$ map can be regarded as a map of combinatorially stratified sets in which each $n$-stratum of therange is a disjoint union of copies of $R^{n}$. Here we prove the analogous theorem for a smooth map $f: M \rightarrow N$ between smooth manifolds.

An essentially similar (but simplified, since 1.1 is obvious) version of our proof would also apply to $P L$ maps between $P L$ manifolds, so our main theorem applies in the $P L$ category as well. The theorem will be used elsewhere [2] to show that Cohen's notion of transverse cellularity [1] may be applied in the smooth category as well.

Let $N$ be a smooth $n$-manifold imbedded in some high-dimensional Euclidean space $R^{N}$. An imbedding $h: X \rightarrow N$ of a simplical complex $X$ into $N$ is called a smooth imbedding if $h^{-1}(\partial N)$ is a subcomplex and, for every $k$-simplex $\sigma$ of $X$, there is a neighborhood $U$ of $h(\sigma)$ in $R^{N}$ and a diffeomorphism $g: U \rightarrow R^{k} \times R^{N-k}$ such that $g h: \sigma \rightarrow R^{k} \times\{0\}$ is the linear map of $\sigma$ onto the standard $k$-simplex $\Delta^{k} \subset R^{k}$. (The use of $R^{N}$ is solely to avoid a special discussion of $\partial N$.) If $X$ is a combinatorial manifold and $h$ is a homeomorphism, then $h$ is called a smooth triangulation of $N$. Combinatorial triangulations of smooth manifolds always exist (see e.g., [4]).

If $f: M \rightarrow N$ is a smooth map of manifolds, a smoothly imbedded complex $h: X \rightarrow N$ is said to be transverse to $f$ over a closed $k$-simplex $\sigma$ in $X$ if the composition $p_{2} g f: M \rightarrow R^{N-k}$ has no critical points near $f^{-1}(h(\sigma))$. In particular, $f^{-1}\left(h\left(\sigma^{\circ}\right)\right)$ is a smooth submanifold of $M$. The definition is independent of the choice of $U, g$, or the imbedding of $N$ in $R^{N}$. Our goal is the proof of

THEOREM 0.1. Let $f: M \rightarrow N$ be a proper smooth map of smooth manifolds, $X \subset N$ a smoothly imbedded simplicial complex, $K \subset X$ a subcomplex of $X$ transverse to $f$.

If $X \cap \partial N \subset K$ or $\partial N$ is transverse to $f$ then there is an ambient diffeotopy $h_{t}: N \rightarrow N$, fixed near $K$, from the identity $h_{0}$ to a map $h_{1}$ such that $h_{1}(X)$ is transverse to $f$. Moreover, the diffeotopy $h_{t}$ 
may be made arbitrarily small in any Riemannian metric on $N$.

In particular, if $f: M \rightarrow N$ is any smooth map between closed smooth manifolds, then $N$ has a smooth triangulation transverse to $f$, and so $f$ may be regarded as a strata preserving map of smooth stratified spaces in which each stratum of the range is a disjoint union of copies of $R^{n}$.

Our result is in fact somewhat stronger: if $\sigma$ is a closed simplex, $f^{-1} h_{1}(\sigma)$ will be a topological manifold with smooth interior and boundary $f^{-1} h(\partial \sigma)$ (see Remark 1.2).

Some notation. Let $r: R^{k} \rightarrow R$ be the map $r\left(x_{1}, \cdots, x_{k}\right)=\left(\Sigma x_{i}^{2}\right)^{1 / 2}$, $\alpha B^{k}=r^{-1}[0, \alpha]$, for $\alpha>0$. If $M$ is a manifold, $M$ denotes interior of $M$. Let $\alpha I^{k}=\alpha \stackrel{\circ}{ }^{1} \times \cdots \times \alpha \dot{B}^{1}$ (k-times). For any $X$, the identity $\operatorname{map} X \rightarrow X$ is denoted $\mathrm{id}_{X}$.

1. Collaring smooth maps near $\partial \Delta^{k}$. Let $\Delta^{k}$ be a $k$-simplex in $R^{k}$ with barycenter at the origin. Suppose $f: V \rightarrow R^{k} \times R^{n}$ is a proper smooth map of smooth manifolds and suppose the complex $\partial \Delta^{k}$ is transverse to $f$.

Proposition 1.1. For some $0<\alpha<1$ and $\varepsilon>0$ there exist

(1) a smooth $m-n-1$ manifold $L$

(2) a diffeomorphism $c: \stackrel{\circ}{B}^{k} \rightarrow \stackrel{\circ}{\Delta}^{k}$

(3) a diffeomorphism

$$
\bar{c}: L \times(\alpha, 1) \times \varepsilon B^{n} \longrightarrow f^{-1}\left[c\left(\dot{B}^{k}-\alpha B^{k}\right) \times \varepsilon B^{n}\right]
$$

such that

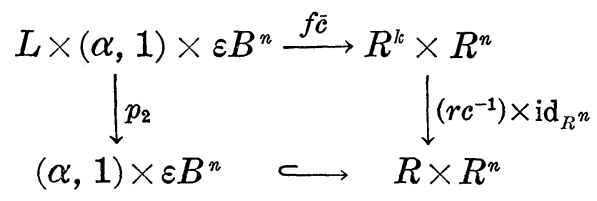

commutes.

Proof. Case 1: $n=0$.

Proof of case 1: Let $w: R^{k} \rightarrow \tau_{R^{k}}$ be the smooth vector field $\operatorname{grad}(r)$. The trajectories of $w$ are the rays from the origin, so, since $\Delta^{k}$ is convex, there is a unique trajectory through each point of $\partial \Delta^{k}$.

For each $j$-simplex $\sigma$ of $\partial \Delta^{k}$ let $h_{\sigma}^{0}: R^{j} \rightarrow R^{k}$ be a linear imbedd- 
ing such that $\sigma \subset$ image $h_{\sigma}^{0}$. By Picard's theorem, there is a unique smooth map $h_{\sigma}: R^{j} \times R \rightarrow R^{k}$ such that $h_{\sigma} \mid R^{j} \times 0=h_{\sigma}^{0}$ and $d h_{\sigma}$ carries the vector field $\operatorname{grad}\left(p_{2}: R^{j} \times R \rightarrow R\right)$ to $w$. If $\tau$ is a $(k-1)$-simplex then $h_{\tau}$ is an imbedding onto a neighborhood of $\tau$ in $R^{k}$.

Order the $(k-1)$-simplices (faces) $\tau_{0}, \cdots, \tau_{k}$ of $\partial \Delta^{k}$. Any $j$-simplex $\sigma$ of $\partial \Delta^{k}$ is contained in $k-j$ faces $\tau_{i_{1}}, \cdots, \tau_{i_{k-j}}, i_{l}<i_{l+1}$. Define the map $q_{i_{l}}$ from a neighborhood of $\tau_{i_{l}}$ to $R$ by $q_{i_{l}}=p_{2} h_{\tau_{i_{l}}}^{-1}$ and on a neighborhood $U_{\sigma}$ of $\sigma$ define $q_{\sigma}: U_{\sigma} \rightarrow R^{k-j}$ by $\left(q_{\sigma}\right)_{l}=q_{i_{l}}$. Observe:

(1) Since $f$ is transverse to $\partial \Delta^{k}$ we may choose $U_{\sigma}$ so small that $q_{\sigma} f: V \rightarrow R^{k-j}$ has no critical values near 0 in $R^{k-j}$.

(2) $d q_{o}(w)=\operatorname{grad}\left(p_{1}+\cdots+p_{k-j}\right)$ where $p_{i}: R^{k-j} \rightarrow R$ is projection on the $i$ th factor.

(3) If $\sigma \subset \sigma^{\prime}$, then $q_{\sigma^{\prime}} \mid U_{\sigma}$ is just $q_{\sigma}$ followed by a projection.

Claim. There is a smooth vector field $v$ on $V$ near $f^{-1}\left(\partial \Delta^{k}\right)$ such that for every $j$-simplex $\sigma$ of $\partial \Delta^{k}$, and $y$ sufficiently close to $f^{-1}(\sigma), d\left(q_{\sigma} f\right)(v(y))=d\left(q_{\sigma}\right)(w(f(y)))$.

Proof of claim. Use induction over simplices. Suppose $v$ has been defined near $f^{-1}((j-1)$-skeleton $), 0 \leqq j \leqq k-1$. Let $\sigma$ be a $j$-simplex. Then, by (2) and (3) above, $d\left(q_{\sigma} f\right)(v)=d q_{\sigma}(w)=\operatorname{grad}$ $\left(p_{1}+\cdots+p_{k-j}\right)$ near $f^{-1}(\partial \sigma)$. By $(1)$ there is a vector field $v_{\sigma}$ defined on $f^{-1}\left(U_{\sigma}\right)$ such that $d\left(q_{\sigma} f\right)\left(v_{\sigma}\right)=\operatorname{grad}\left(p_{1}+\cdots+p_{k-j}\right)$. Let $\varphi: f^{-1}\left(U_{\sigma}\right) \rightarrow$ $[0,1]$ be a smooth map with support where $v$ is defined and such that $\varphi=1$ near $f^{-1}(\partial \sigma)$. Then $\varphi v+(1-\varphi) v_{\sigma}$ is an appropriate extension of $v$ near $\sigma$, completing the inductive step and so verifying the claim.

Now choose $\gamma>0$ so small that for any $j$-simplex $\sigma$ of $\partial \Delta^{k}$, $q_{\sigma}^{-1}\left(\gamma I^{k-j}\right)$ is contained in $U_{\sigma}, q_{\sigma}: U_{\sigma} \rightarrow R^{k-j}$ is nonsingular over $\gamma I^{k-j}$ and $v$ is defined on $\left(q_{\sigma} f\right)^{-1}\left(\gamma I^{k-j}\right)$. Without loss of generality, let $U_{\sigma}=q_{\sigma}^{-1}\left(\gamma I^{k-j}\right)$, so $d\left(q_{\sigma} f\right)(v(y))=d\left(q_{\sigma}\right)(w(f(y)))$ throughout $f^{-1}\left(U_{\sigma}\right)$.

Let $\mu: R \rightarrow[-1,1]$ be a smooth map such that $\mu(x)=-x /|x|$ for $|x| \geqq \gamma / 2, \mu(x)=-x$ for $x$ near 0 , and $\mu(-\gamma / 2, \gamma / 2) \rightarrow(-1,1)$ is a diffeomorphism. Define $\rho_{k-j}: R^{k-j} \rightarrow R$ by $\rho_{k-j}\left(x_{1}, \cdots, x_{k-j}\right)=$ $\prod_{i=1}^{k-j} \mu\left(x_{i}\right)$. For each $j$-simplex $\sigma$ of $\partial \Delta^{k}$, define $\rho_{\sigma}=\rho_{k-j} q_{\sigma}: U_{\sigma} \rightarrow R$. Notice that if $\sigma \subset \sigma^{\prime}$, then $\rho_{\sigma}$ coincides with $\rho_{\sigma^{\prime}}$ except perhaps where $\left|q_{\sigma_{i}}(x)\right|<\gamma / 2$, some $0<i<k-j$. In particular $\rho_{\sigma}$ coincides with $\rho_{\sigma^{\prime}}$ except well within $U_{\sigma}$. We may therefore consistently define a smooth $\rho: R^{k} \rightarrow[0,1]$ as follows. Let $U=\bigcup_{o} U_{\sigma}, \sigma$ in $\partial \Delta^{k}$, and let $\sigma(x)$ denote the lowest dimensional simplex of $\partial \Delta^{k}$ for which $U_{\sigma(x)}$ contains $x$.

(i) If $x$ is in $\Delta^{k}-U$, let $\rho(x)=1$

(ii) If $x$ is in $R^{k}-\left(\Delta^{k} \cup U\right)$, let $\rho(x)=-1$ 
(iii) If $x$ is in $U, \rho(x)=\rho_{\sigma(x)} x$.

Clearly $\rho^{-1}(0)=\partial \Delta^{k}$ and $\rho \mid \Delta^{k}>0$. Define new vector fields $w^{\prime}$ and $v^{\prime}$ over $R^{k}$ and $f^{-1}(U)$ by $w^{\prime}(x)=\rho(x) w(x), v^{\prime}(y)=\rho(f(y)) v(y)$ for $x$ in $R^{k}$ and $y$ in $f^{-1}(U)$. Just as for $w$ and $v$ we have, for any $j$-simplex $\sigma$ of $\partial \Delta^{k}$ and $y$ in $f^{-1}(U), d\left(q_{\sigma} f\right)\left(v^{\prime}(y)\right)=d q_{\sigma}\left(w^{\prime}(f(y))\right)$. In other words, suppose we define the vector field $u_{\sigma}$ in $R^{k-j}$ by $u_{o}=\rho_{k-j} \operatorname{grad}\left(p_{1}+\cdots+p_{k-j}\right)$. Then, in fact, we have $d\left(q_{\sigma} f\right)\left(v^{\prime}(y)\right)=$ $d q_{\sigma}\left(w^{\prime}(f(y))\right)=u_{\sigma}\left(q_{\sigma} f(y)\right)$.

Choose $\varepsilon>0$ so small that any point $s$ in $\rho^{-1}(\varepsilon)$ satisfies $q_{\tau}(s)>-\gamma / 2$ for some face $\tau$ of $\partial \Delta^{k}$. Then for $\sigma=\sigma(s)$,

$$
\begin{aligned}
& d\left(\rho_{k-j}\right)\left(u_{\sigma}\left(q_{\sigma}(s)\right)\right)=\rho_{k-j}\left(q_{\sigma}(s)\right) \cdot \sum_{i=1}^{k-j} \frac{\partial \rho_{k-j}}{d x_{i}} \\
& =\rho_{k-j} \cdot\left(\sum_{i=1}^{k-j} \frac{\mu^{\prime}\left(q_{\sigma_{i}}\right)}{\mu\left(q_{\sigma_{i}}\right)}\right) \cdot \prod_{i=1}^{k-j} \mu\left(q_{\sigma_{i}}\right)=\left(\sum_{i=1}^{k-j} \frac{\mu^{\prime}\left(q_{\sigma_{i}}\right)}{\mu\left(q_{\sigma_{i}}\right)}\right) \rho_{k-j}^{2}<0
\end{aligned}
$$

since $\mu\left(q_{\sigma_{i}}\right)>0$ in $\dot{\Delta}^{k}, \mu^{\prime}\left(q_{\sigma_{i}}\right) \leqq 0$ and for at least one term (where $\left.q_{\sigma_{i}}=q_{\tau}\right), \mu^{\prime}\left(q_{\sigma_{i}}\right)<0$. Then, for $f(y)=s, d(\rho f) v^{\prime}(y)=(d \rho) w^{\prime}(s)=$ $d\left(\rho_{k-j} q_{\sigma}\right) w(s)=d\left(\rho_{k-j}\right) U_{\sigma}\left(q_{\sigma}(s)\right)<0$, so $\rho$ and $\rho f$ are transverse to $\varepsilon$. Define $S$ and $L$ to be the smooth suqmanifolds $\rho^{-1}(\varepsilon)$ and $f^{-1}(S)$ of $\Delta^{k}$ and $V$ respectively. Since $(d \rho) w^{\prime}<0$ at all $s$ in $S=\rho^{-1}(\varepsilon)$, each trajectory of $w^{\prime}$ intersects $S$ precisely once. Similarly, each trajectory of $v^{\prime}$ intersects $L$ precisely once.

Picard's theorem then provides smooth imbeddings $c_{0}: S \times[0, \infty) \rightarrow$ $\dot{\Delta}^{k}$ and $\bar{c}_{0}: L \times[0, \infty) \rightarrow f^{-1}\left(\stackrel{\circ}{ }^{k}\right)$ whose trajectories $c_{0}(s \times[0, \infty))$ and $\bar{c}_{0}(l \times[0, \infty))$ have tangent vectors $w^{\prime}$ and $v^{\prime}$ respectively.

Claim 2. Diagram A below commutes.

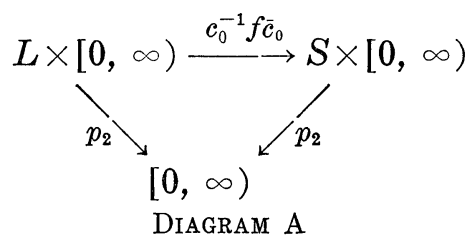

Proof of Claim 2. Unfortunately, $f_{*}\left(v^{\prime}\right) \neq w^{\prime}$, so the proof is not immediate. For $\left(l, t_{0}\right)$ in $L \times[0, \infty), \sigma$ any $j$-simplex in $\partial \Delta_{k}$, let $*_{\sigma}$ denote the following condition:

$l$ has a neighborhood $\widetilde{L}$ in $L$ such that for some codimension 1 manifold $\widetilde{M}$ in $R^{k-j}$ transverse to $u_{k-j}, c_{0}\left(\widetilde{L} \times t_{0}\right) \subset\left(q_{\sigma} f\right)^{-1}(\widetilde{M})$.

Then notice:

(a) If $\sigma=\sigma\left(f\left(\bar{c}_{0}\left(l, t_{0}\right)\right)\right)$, so $d\left(q_{\sigma} f\right) v^{\prime}=u_{\sigma}$, then the unit flow of $\widetilde{L} \times t_{0}$ in $L \times[0, \infty)$ is mapped by $q_{o} f \bar{c}_{0}$ to the flow of $\tilde{M}$ along $u_{k-j}$, so $*_{\sigma}$ will continue to hold for $t \geqq t_{0}$ as long as $\sigma=\sigma\left(f\left(\bar{c}_{0}(l, t)\right)\right)$. 
(b) If $\sigma$ is a simplex in $\sigma^{\prime} \subset \partial \Delta^{k}$, then $*_{\sigma}$ holds wherever $\rho_{\sigma}=\rho_{\sigma^{\prime}}$, i.e., except well within $U_{\sigma}$. Indeed, $q_{\sigma}$ is just $q_{\sigma^{\prime}}$ composed with a projection $p$. Let $\widetilde{M}=p^{-1}\left(\widetilde{M}^{\prime}\right)$, where $\widetilde{M}^{\prime}$ is the manifold of condition $*_{\sigma^{\prime}}$. Since we assume $\rho_{\sigma^{\prime}}=\rho_{\sigma}, d p\left(u_{\sigma}\right)=u_{\sigma^{\prime}}$ so $u_{\sigma}$ is transverse to $\tilde{M}$.

(c) If $\sigma=\sigma(f \bar{c}(l, 0))$, then $*_{\sigma}$ holds for $(l, 0)$. Indeed, take $\widetilde{M}$ to be $\rho_{k-j}^{-1}(\varepsilon)$; we showed above that $u_{\sigma}$ is transverse to $\tilde{M}$ and defined $L$ so that it coincides with $\left(q_{\sigma} f\right)^{-1}(\widetilde{M})$ near $\bar{c}(l, 0)$.

(d) Since the trajectories of $u_{\sigma}$ never increase their distance from $0 \in R^{k-j}$ it follows that if $\sigma=\sigma\left(f \bar{c}_{0}\left(l, t_{0}\right)\right)$, then $\sigma\left(f \bar{c}_{0}(l, t)\right) \subset \sigma$ for all $t \geqq t_{0}$.

Combining $a-d$, it follows that condition $*_{\sigma}$ holds for any $(l, t)$ when $\sigma=\sigma\left(f \bar{c}_{0}(l, t)\right)$.

Now, by definition, $A$ commutes over $0 \in[0, \infty)$. The set of values $t \in[0, \infty)$ over which $A$ commutes is clearly closed; we show that is also open. Let $t_{0}$ be a point such that $c_{0}^{-1} f \bar{c}_{0}\left(L \times t_{0}\right)=S \times t_{0}$. Choose any $l$ in $L$ and let $\sigma=\sigma\left(f \bar{c}_{0}\left(l, t_{0}\right)\right)$ be a $j$-simplex.

Then there is a neighborhood $\widetilde{L}$ of $l$ and a codimension one manifold $\tilde{M}$ of $R_{k-j}$ transverse to $u_{o}$ such that $\bar{c}_{0}\left(\tilde{L} \times t_{0}\right) \subset\left(q_{0} f\right)^{-1}(\tilde{M})$. Then $f^{-1}(\widetilde{M})$ contains a neighborhood $\widetilde{S}$ of $f \bar{c}_{0}\left(l, t_{0}\right)$ in $S \times t_{0}$, but since $d q_{\sigma}\left(w^{\prime}\right)=u_{\sigma}$, the unit upward flow of $\widetilde{S}$ in $S \times[0, \infty)$ is mapped by $q_{o} c_{0}$ to the unit upward flow of $\widetilde{M}$ under $u_{\sigma}$. By condition (a) above, the unit upward flow of $\widetilde{L}$ in $L \times[0, \infty)$ then is mapped by $c_{0}^{-1} f \bar{c}_{0}$ to the unit upward flow of $c_{0}^{-1} f \bar{c}_{0}(\widetilde{L})$ in $S \times[0, \infty)$. Hence $A$ commutes near $\left(l, t_{0}\right)$. Since $f$ is proper, $L$ is compact. Thus a repetition of our argument near a finite number of points $l$, shows $A$ commutes over a neighborhood of $t_{0}$. Hence $A$ commutes everywhere, verifying Claim 2.

It remains only to show that $c$ extends to an imbedding of $\dot{B}^{k}$ in $\dot{\Delta}^{k}$. Each trajectory of $w^{\prime}$ intersects both $S \times 0$ and the boundary of a small ball about 0 in $\Delta^{k}$ exactly once. It is then a classical result that the ball can be smoothly deformed so that the interior of a collar of its boundary coincides with $S \times(0, \infty)$, giving an extension of $c$ over the rest of $\Delta^{k}$.

Case 2. $n>0$.

Proof of Case 2. Since $f$ is transverse to $\partial \Delta^{k}$ the map $p_{2} f: V \rightarrow$ $R^{n}$ is transverse to 0 near $\partial \Delta^{k}$. Then there is a neighborhood $U$ of $\partial \Delta^{k}$ in $R^{k}$ such that $f^{-1}(U)$ is a smooth submanifold of $V$, and $f \mid f^{-1}(U) \rightarrow U$ is transverse to $\partial \Delta^{k}$. Apply Case 1 to get $\bar{c}: L \times(0,1) \rightarrow f^{-1}(U)$, $c: S \times(0,1) \rightarrow U$ such that $c^{-1} f \bar{c}$ commutes with projection to $(0,1)$. Extend $c$ to an imbedding $c: S \times(0,1) \times R^{n} \rightarrow U \times R^{n}$ by crossing with $\mathrm{id}_{R^{n}}$. 
Since $c^{-1} f$ is transverse to $S \times(0,1)$, it follows from classical tubular neighborhood theory that $\bar{c}$ extends to a map $c: L \times(0,1) \times$ $\varepsilon B^{n} \rightarrow V$ such that $c^{-1} f \bar{c}$ commutes with projection to $(0,1) \times \varepsilon B^{n}$.

REMARK 1.2. Since each trajectory of $w^{\prime}$ (resp. $v^{\prime}$ ) lies in a trajectory of $w$ (resp. $v$ ) and each point of $\partial \Delta^{k}$ (resp. $\left.f^{-1}\left(\partial \Delta^{k}\right)\right)$ lies in a unique trajectory of $w$ (resp. $v$ ), each point of $\partial \Delta^{k}\left(\operatorname{resp} . f^{-1}\left(\partial \Delta^{k}\right)\right.$ ) is the limit point of a unique trajectory of $w^{\prime}$ (resp. $v^{\prime}$ ). Therefore the smooth imbeddings $c: S \times[0,1) \rightarrow \Delta^{k}, \bar{c}: L \times[0,1) \rightarrow f^{-1}\left(\Delta^{k}\right)$ given by $c(s, t)=c_{0}(s, t / 1-t), \bar{c}(l, t)=\bar{c}_{0}(l, t / 1-t)$ extend to topological collars $c: S \times[0,1] \rightarrow \Delta^{k}$ and $\bar{c}: L \times[0,1] \rightarrow f^{-1}\left(\Delta^{k}\right)$ of $\partial \Delta^{k}$ and $f^{-1}\left(\partial \Delta^{k}\right)$ respectively.

2. Proof of the theorem. First consider the following special case.

LEMma 2.1. Let $f: M \rightarrow R^{k} \times R^{n}$ be a proper smooth map transverse to $\partial \Delta^{k}=\partial \Delta^{k} \times 0 \subset R^{k} \times 0$ and let $\delta: R^{k} \times R^{n} \rightarrow(0, \infty)$ be continuous. Then there is an ambient diffeotopy $h_{t}: R^{k} \times R^{n} \rightarrow R^{k} \times R^{n}$, fixed outside a compact set in $\dot{\Delta}^{k} \times R^{n}$, from the identity $h_{0}$ to a map $h_{1}$ such that $h_{1}\left(\Delta^{k}\right)$ is transverse to $f$. Furthermore $d\left(h_{t}(x), x\right)<\delta(x)$, $0 \leqq t \leqq 1$.

Proof. Sinee $h_{t}$ will be fixed outside a compactum, we may assume $\delta$ is constant.

Let $\varepsilon, L, c, \bar{c}, \alpha$ be as in 1.1. With no loss of generality, let $\varepsilon=\delta$. Since critial values of $p_{2} f: M \rightarrow R^{n}$ are meager, by Sard's theorem, there is a regular value $y_{0}$ in $\varepsilon \dot{B}^{n}$. Let $\psi_{t}, 0 \leqq t \leqq 1$ be a diffeotopy of id $R^{n}$ with support in $\varepsilon \dot{B}^{n}$ carrying 0 to $y_{0}$. Let $\mu: R \rightarrow[0,1]$ be a smooth map such that $\mu(x)=1$ for $x$ near $(-\infty, \alpha], \mu(x)=0$ for $x$ near $[1, \infty)$. Define

$$
h_{t}: c\left(B^{k}\right) \times R^{n} \longrightarrow c\left(B^{k}\right) \times R^{n}
$$

by $h_{t}(c(x), y)=\left(c(x), \psi_{t /(|x|)}(y)\right)$. Extend $h_{t}$ by the identity to the rest of $R^{k} \times R^{n}$.

We claim $h_{1}\left(\Delta^{k}\right)$ is transverse to $f$. Certainly $h_{1} c\left(\alpha B^{k}\right)$ is transverse to $f$, for $h_{1} c\left(\alpha B^{k}\right)=\alpha B_{k} \times \psi_{1}(y)=\alpha B_{k} \times y_{0}$. Since $\mu(|x|)=0$ for $|x|$ near $1, h$ is fixed near $\partial \Delta^{k}$, so, by hypothesis, $h_{1}\left(\Delta^{k}\right)$ is transverse to $f$ near $\partial \Delta^{k}$.

Define $\bar{h}: \bar{c}\left(L \times(\alpha, 1) \times \varepsilon B^{n}\right) \rightarrow \bar{c}\left(L \times(\alpha, 1) \times \varepsilon B^{n}\right)$ by $\bar{h}(\bar{c}(l, r, y))=$ $\bar{c}\left(l, r, \psi_{\mu(r)}(y)\right)$. Then $h_{1}^{-1} f \bar{h}=f$, which is transverse to $c\left(B^{k}-\alpha B^{k}\right)$, by 1.1. Since $\bar{h}$ is a diffeomorphism, $h_{1}^{-1} f$ is also transverse to $c\left(\dot{B}^{k}-\alpha B^{k}\right)$, so $f$ is transverse to $h_{1} c\left(\stackrel{\circ}{B}^{k}-\alpha B^{k}\right)$, completing the proof. 
Proof of 0.1 .

Case 1: $X \cap \partial N \subset K$.

The proof is a straightforward induction over simplices of $X-K$; suppose $h_{t}$ has been constructed so that $f$ is now transverse to the $(k-1)$-skeleton. Apply 2.1 to a neighborhood of each $k$-simplex, the neighborhoods chosen so that $f$ is already transverse to the $k$-simplices wherever neighborhoods overlap. This completes the inductive step, hence the proof in this case.

Case 2. $\partial N$ is transverse to $f$.

Apply Case 1 first to $f \mid f^{-1}(\partial N)$ isotoping $\partial N$ until the subcomplex $X \cap \partial N$ is transverse to $f \mid f^{-1}(\partial N)$. Extend to an isotopy of $N$. Since $f$ is transverse to $\partial N$, after the isotopy $f$ will be transverse to $X \cap \partial N$. This reduces the problem to the previous case.

\section{REFERENCES}

1. M. M. Cohen, Simplical structures and transverse cellularity, Ann. of Math., 85 (1967), 218-245.

2. M. Scharlemann, Approximating smooth CE maps, in Proceedings of the 1977 Santa Barbara Topology Conference, (to appear).

3. L. C. Siebenmann, Deformation of homeomorphisms on stratified sets, Comm. Math. Helv., 47 (1972), 123-163.

4. J. H. C. Whitehead, On $C^{1}$ complexes, Ann. of Math., 41 (1940), 804-824.

Received December 29, 1977. Supported in part by an NSF Grant.

UNIVERSITY OF CALIFORNIA

SANTA BARBARA, CA 93106 



\section{PACIFIC JOURNAL OF MATHEMATICS}

\section{EDITORS}

RICHARD ARENS (Managing Editor)

University of California

Los Angeles, California 90024

C. W. CURTIS

University of Oregon

Eugene, OR 97403

C. C. MOORE

University of California

Berkeley, CA 94720
J. DugundjI

Department of Mathematics University of Southern Californı Los Angeles, California 90007

R. Finn and J. Milgram Stanford University Stanford, California 94305

ASSOCIATE EDITORS

E. F. BECKENBACH

B. H. NeUmanN

F. WOLF

K. YoSHIDA

\section{SUPPORTING INSTITUTIONS}

UNIVERSITY OF BRITISH COLUMBIA CALIFORNIA INSTITUTE OF TECHNOLOGY

UNIVERSITY OF CALIFORNIA

MONTANA STATE UNIVERSITY

UNIVERSITY OF NEVADA, RENO

NEW MEXICO STATE UNIVERSITY

OREGON STATE UNIVERSITY

UNIVERSITY OF OREGON
UNIVERSITY OF SOUTHERN CALIFORNIA

STANFORD UNIVERSITY

UNIVERSITY OF HAWAII

UNIVERSITY OF TOKYO

UNIVERSITY OF UTAH

WASHINGTON STATE UNIVERSITY

UNIVERSITY OF WASHINGTON 


\section{Pacific Journal of Mathematics}

\section{Vol. 80, No. $1 \quad$ September, 1979}

Jeroen Bruijning and Jun-iti Nagata, A characterization of covering dimension by

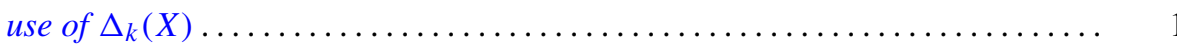

John J. Buoni and Albert Jonathan Klein, On the generalized Calkin algebra ...... 9

Thomas Ashland Chapman, Homotopy conditions which detect simple homotopy



John Albert Chatfield, Solution for an integral equation with continuous interval

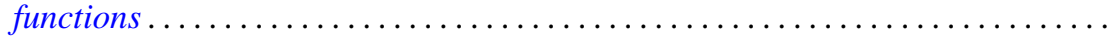

Ajit Kaur Chilana and Ajay Kumar, Spectral synthesis in Segal algebras on

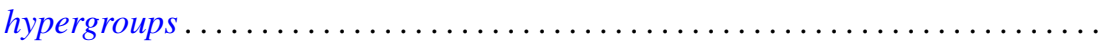

Lung O. Chung, Jiang Luh and Anthony N. Richoux, Derivations and

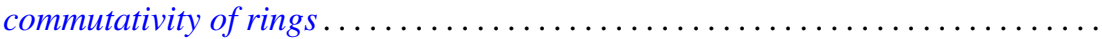

Michael George Cowling and Paul Rodway, Restrictions of certain function spaces to closed subgroups of locally compact groups .....................

David Dixon, The fundamental divisor of normal double points of surfaces........

Hans Georg Feichtinger, Colin C. Graham and Eric Howard Lakien,

Nonfactorization in commutative, weakly selfadjoint Banach algebras . . . . . . .

Michael Freedman, Cancelling 1-handles and some topological imbeddings ....... .

Frank E., III Gerth, The Iwasawa invariant $\mu$ for quadratic fields . . . . . . . . . . . . . .

Maurice Gilmore, Three-dimensional open books constructed from the identity

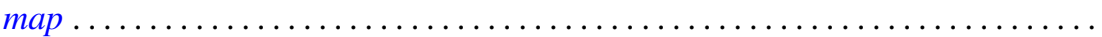

Stanley P. Gudder, A Radon-Nikodým theorem for $*$-algebras .

Peter Wamer Harley, III and George Frank McNulty, When is a point Borel? .

Charles Henry Heiberg, Fourier series with bounded convolution powers . .

Rebecca A. Herb, Characters of averaged discrete series on semisimple real Lie

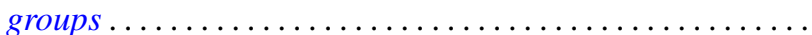

Hideo Imai, On singular indices of rotation free densities . .

Sushil Jajodia, On 2-dimensional CW-complexes with a single 2-cell . . .

Herbert Meyer Kamowitz, Compact operators of the form $u C_{\varphi}$

Matthew Liu and Billy E. Rhoades, Some properties of the Chebyshev method...

213

George Edgar Parker, Semigroups of continuous transformations and generating



Samuel Murray Rankin, III, Oscillation results for a nonhomogeneous

equation ...

Martin Scharlemann, Transverse Whitehead triangulations ...

Gary Joseph Sherman, A lower bound for the number of conjugacy classes in a

finite nilpotent group

Richard Arthur Shoop, The Lebesgue constants for $\left(f, d_{n}\right)$-summability .

Stuart Jay Sidney, Functions which operate on the real part of a uniform



Tim Eden Traynor, The group-valued Lebesgue decomposition 